\title{
Spacetimes with continuous linear isotropies II: boosts
}

\author{
M. A. H. MacCallum ${ }^{1}$
}

Received: 27 April 2021 / Accepted: 18 May 2021 / Published online: 22 June 2021

(C) The Author(s) 2021

\begin{abstract}
Conditions are found which ensure that local boost invariance (LBI), invariance under a linear boost isotropy, implies local boost symmetry (LBS), i.e. the existence of a local group of motions such that for every point $P$ in a neighbourhood there is a boost leaving $P$ fixed. It is shown that for Petrov type D spacetimes this requires LBI of the Riemann tensor and its first derivative. That is also true for most conformally flat spacetimes, but those with Ricci tensors of Segre type [1(11,1)] may require LBI of the first three derivatives of curvature to ensure LBS.
\end{abstract}

Keywords Spacetime symmetries · Cartan invariants · Equivalence problem

\section{Contents}

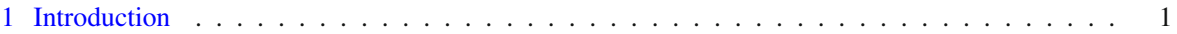

2 Petrov type D spacetimes with local boost invariance . . . . . . . . . . . . . . . . 3

3 Conformally flat spacetimes with local boost invariance . . . . . . . . . . . . . . . . . . . 6

3.1 The first derivatives and Bianchi identities . . . . . . . . . . . . . . . . 6

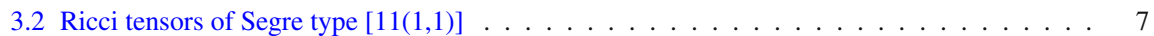

3.3 Ricci tensors of Segre type $[(11)(1,1)] \ldots \ldots \ldots \ldots \ldots \ldots \ldots$

3.4 Ricci tensors of Segre type $[1(11,1)] \ldots \ldots \ldots \ldots \ldots \ldots \ldots$

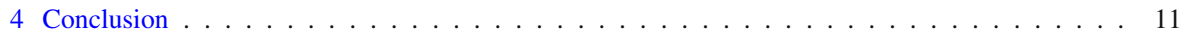

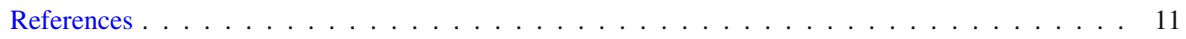

\section{Introduction}

In the first paper of this series [9] spacetimes with a local spatial rotational invariance were (re)-investigated. Here the corresponding issues for local boost invariance are studied. The issues arising and the methods to be used are set out in Sections 1 and 2

M. A. H. MacCallum

M.A.H.MacCallum@qmul.ac.uk

1 School of Mathematical Sciences, Queen Mary University of London, Mile End Road, London E1 $4 \mathrm{NS}, \mathrm{UK}$ 
of the previous paper. Only those key points required to make this paper reasonably self-contained will be repeated here.

In studying local rotational symmetry of spacetime (LRS), Ellis [2] introduced three definitions which he showed to be equivalent for dust spacetimes. The results were later extended to spacetimes with a perfect fluid and electromagnetic field [15]. Here Ellis's definition $\left(\mathrm{A}_{m}\right)$ will be studied. It reads:

$\left(\mathrm{A}_{m}\right)$ At each point $P$ in an open neighbourhood $U$ of a point $P_{o}$, there exists a nondiscrete subgroup $g$ of the Lorentz group in the tangent space $T_{P}$ which leaves invariant the curvature tensor and all its covariant derivatives to the $m$-th order.

Implicit in this definition are conditions on the smoothness of the manifold and the correspondence between the $g$ at separated points. This paper considers the case where the group $g$ of linear isotropies contains boosts. This is called local boost invariance (LBI), 'local' meaning that the same $g$ applies throughout $U$. 'Spacetime' here just means a four-dimensional Lorentzian manifold. The field equations of general relativity will not be used, but in cases where the Ricci tensor takes the form that would be implied by specific matter content in general relativity that interpretation is referred to.

The starting conjecture (based on a claim by Siklos [13] now known to be false in general) is that $\left(A_{1}\right)$ is sufficent to imply Ellis's definition $(C)$, i.e.

There exists a local group of motions $G_{r}$ in an open neighbourhood $W$ of a point $P_{o}$ which is multiply transitive on some $q$ [-dimensional] surface through each point $P$ of $W$.

With LBI this would imply that the spacetime had local boost symmetry (LBS), i.e. that the group $G_{r}$ contained, for every $P \in W$, a subgroup of boosts leaving $P$ fixed. A theorem of Hall [5] implies, under appropriate topological and smoothness conditions, that $W$ is a region of a manifold in which the same $G_{r}$ acts globally.

Only Petrov type D and conformally flat spacetimes have a Weyl tensor that can satisfy $\left(\mathrm{A}_{0}\right)$ (or $\left(\mathrm{A}_{m}\right)$ for larger $m$ ) with a group $g$ containing boosts. There are rather more Ricci tensor types that can satisfy $\left(\mathrm{A}_{0}\right)$ for boosts. For $\left(\mathrm{A}_{0}\right)$ to apply to the whole Riemann tensor, the Weyl and Ricci tensors must of course be appropriately aligned, and there may be a nonzero Ricci scalar. The tracefree part of the Ricci tensor can be characterized by its Segre type. The possible invariance groups of the Ricci tensor were listed by Segre type in Table 5.2 of Stephani et al. [14]. Table 1 here lists those with nontrivial invariance groups $\hat{I}_{0}$ which include a boost. Cahen and Defrise [1] showed that for Petrov type D spacetimes with boost (or spatial rotation) invariance and any compatible Ricci tensor, $\left(\mathrm{A}_{2}\right)$ was a sufficient criterion for the spacetime to be LRS or LBS. Subsequently Goode and Wainwright [4] gave criteria for the LRS Petrov type $D$ case in terms of the spin coefficients and curvature expressed in a Newman-Penrose (NP) null tetrad. These criteria were shown in MacCallum [9] to be equivalent to $\left(A_{1}\right)$. The discrepancy with Cahen and Defrise's use of $\left(\mathrm{A}_{2}\right)$ is shown in the Appendix of MacCallum [9] to be due to a less suitable choice of frame in the calculations.

Here it is shown in Sect. 2, by arguments parallel to those of Goode and Wainwright [4], that $\left(A_{1}\right)$ is also sufficient for LBS in Petrov type D spacetimes. In Sect. 3, 
Table 1 Nontrivial invariance groups containing boosts, by Ricci tensor type

\begin{tabular}{ll}
\hline Invariance group & Segre type of the Ricci tensor \\
\hline Boosts (only) & {$[11(1,1)]$} \\
Boosts and rotations & {$[(11)(1,1)]$} \\
$S O(2,1):$ three-dimensional Lorentz group & {$[1(11,1)]$} \\
Full Lorentz group & {$[(111,1)]$} \\
\hline
\end{tabular}

the corresponding question for conformally flat spacetimes is studied. In both these sections the detailed arguments are closely related to those of MacCallum [9] by the asterisk operation of the GHP formalism [3]. A recent preprint addresses local boost invariance of higher dimensional manifolds [11].

As in the previous paper, the Cartan-Karlhede procedure for characterizing spacetimes and testing their equivalence, as outlined in Section 2 of MacCallum [9], is used. It relies on the computation of "Cartan invariants", the components of the Riemann tensor and its covariant derivatives in canonically chosen frames. The implementation used here employs the Newman-Penrose formalism as set out in Chapter 7 of Stephani et al. [14]. The "Newman-Penrose equations" (the Ricci equations) and Bianchi identities [(7.21a)-(7.21r) and (7.32a)-(7.32k) in Stephani et al. [14]] will be referred to below as (NPa)-(NPr) and (Ba)-(Bk).

A minimal set of Cartan invariants sufficient for the above procedure, was defined by MacCallum and Åman [10]. It consists of totally symmetrized spinor derivatives of the Newman-Penrose curvature quantities. Here the shorthand notation for such spinors, as defined in MacCallum [9], will be used. If $Q^{A B C \ldots} E^{\prime} F^{\prime} \ldots$ is a relevant curvature quantity then the notation $Q_{A B^{\prime}}$ denotes the component of $Q^{(A B C \ldots)}{ }_{\left(E^{\prime} F^{\prime} \ldots\right)}$ in which $A$ of the $m$ unprimed indices and $B$ of the $n$ primed indices are contracted with the basis spinors $\iota$ and $\iota$ respectively (and the others with the basis spinors $o$ and $\bar{o}) . \chi$ is said to have valence $(m, n)$. The set defined in MacCallum and Åman [10] consists of the totally symmetrized derivatives of $\Psi, \Phi$ and $\Lambda$, together with, at order $1, \Xi_{D E F W^{\prime}}=\nabla^{C}{ }_{W^{\prime}} \Psi_{C D E F}$ and at order $q+2$, the d'Alembertians of quantities at order $q$. For a totally-symmetrized spinor of valence $(m, n)$, only components with $2(A+B)=m+n$ are LBI.

\section{Petrov type D spacetimes with local boost invariance}

In the calculations, the boost invariance is assumed to act in the $(\boldsymbol{k}, \boldsymbol{l})$ plane of a Newman-Penrose tetrad adapted to the Petrov type D Weyl tensor and to leave the Riemann tensor and its first derivative unchanged ${ }^{1} . \Lambda$ and $\Psi_{2}(\neq 0)$ are boost invariant, so from $\left(\mathrm{A}_{1}\right) D \Lambda=\Delta \Lambda=D \Psi_{2}=\Delta \Psi_{2}=0$. In $\Phi_{A B^{\prime}}$ only the components $\Phi_{11^{\prime}}$ and $\Phi_{02^{\prime}}$ can be nonzero. Boost invariance of the Cartan invariants $\nabla \Psi_{A B^{\prime}}$ requires that

\footnotetext{
${ }_{1}^{1}$ The results however are independent of the choice of frame: they would simply be more difficult to check in a randomly chosen frame.
} 


$$
\nabla \Psi_{01^{\prime}}=\nabla \Psi_{11^{\prime}}=\nabla \Psi_{20^{\prime}}=\nabla \Psi_{31^{\prime}}=\nabla \Psi_{40^{\prime}}=\nabla \Psi_{41^{\prime}}=0
$$

and thus

$$
\kappa=\sigma=\rho=\mu=\lambda=\nu=0 .
$$

These spacetimes are members of Kundt's class.

Therefore, in Petrov type D, invariance of the Riemann tensor and its first derivatives under a boost implies that there is a Newman-Penrose tetrad (a canonical one for Petrov type D, fixed up to a spatial rotation and boost) in which the following criteria hold.

$$
\begin{aligned}
& \left(\mathrm{C}^{\dagger}\right): \kappa=\sigma=\rho=\mu=\lambda=v=0 \\
& \left(\mathrm{C}^{\dagger}\right): \Phi_{01}=\Phi_{12}=0 . \\
& \left(\mathrm{C}^{\dagger}\right): \Delta \Lambda=D \Lambda=0 .
\end{aligned}
$$

[The $\left(\mathrm{Cn}^{\dagger}\right)$ notation is adopted to emphasize the parallel with the conditions $(\mathrm{C} 1)-(\mathrm{C} 3)$ of Goode and Wainwright [4].] The theorem analogous to Theorem 2.1 of Goode and Wainwright is:

Theorem 1 A space-time (assumed conformally curved) is LBS if and only if there exists a null tetrad $(\boldsymbol{k}, \boldsymbol{l}, \boldsymbol{m}, \overline{\boldsymbol{m}})$ in which $\left(\mathrm{C}^{\dagger}\right)-\left(\mathrm{C} 3^{\dagger}\right)$ hold.

To show that conversely $\left(\mathrm{Cl}^{\dagger}\right)-\left(\mathrm{C}^{\dagger}\right)$ imply that the Riemann tensor and its first derivatives are boost invariant, one first inserts $\left(\mathrm{Cl}^{\dagger}\right)$ into ( $\mathrm{NPa}, \mathrm{b}, \mathrm{j}, \mathrm{k}, \mathrm{m}$ and $\left.\mathrm{n}\right)$, and, using $\left(\mathrm{C}^{\dagger}\right)$, obtains that

$$
\Phi_{00^{\prime}}=\Phi_{22^{\prime}}=0, \quad \Psi_{0}=\Psi_{1}=\Psi_{3}=\Psi_{4}=0,
$$

so that the Riemann tensor is Petrov type $\mathrm{D}$ or conformally flat and is boost invariant. (Be) and (Bf) give $D \Psi_{2}=\Delta \Psi_{2}=0$. From above, $\left(\mathrm{Cl}^{\dagger}\right)$ and $\left(\mathrm{C}^{\dagger}\right)$ then guarantee that $\nabla \Lambda$ and $\nabla \Psi_{A B^{\prime}}$ are boost invariant.

Using $\left(\mathrm{Cl}^{\dagger}\right)-\left(\mathrm{C}^{\dagger}\right)$ in $(\mathrm{Bi})$ and $(\mathrm{Bk})$ gives $D \Phi_{11^{\prime}}=\Delta \Phi_{11^{\prime}}=0$, so the gradient of $\Phi_{11^{\prime}}$ is boost invariant. The components of $\nabla \Phi$ that must vanish for LBI are given in full as (3.1)-(3.6) in Sect. 3, and the relations between these components of $\nabla \Phi$ and the Bianchi identities are discussed there. Given $(\mathrm{C} 1)$, they all vanish, due to $(\mathrm{Bb})$ and (Bc), so $\nabla \Phi$ is boost invariant.

To complete the check of the equivalence of the conditions $\left(\mathrm{C}^{\dagger}\right)-\left(\mathrm{C} 3^{\dagger}\right)$ with the assumption that the Riemann tensor and its first derivatives are boost invariant, one has to show that once the remaining frame freedom, a spatial rotation, has been fixed, so that $\Phi_{02^{\prime}}$ is an invariant, $D \Phi_{02^{\prime}}=\Delta \Phi_{02^{\prime}}=0$, which follows if $\varepsilon$ and $\gamma$ are real. (The boost invariance of $\Xi_{A B^{\prime}}$ is readily checked.)

These and other restrictions on the spin coefficients analogous to those in Section 3 of MacCallum [9] are now sought, following analogous steps in Goode and Wainwright [4]. They will enable the LBI of higher derivatives of the curvature to be checked. From $(\mathrm{Bh})$ and $(\mathrm{Bj})$ one finds

$$
\delta\left(-\Psi_{2}-\Phi_{11^{\prime}}+\Lambda\right)=-3 \tau \Psi_{2}+2 \bar{\pi} \Phi_{11}+\pi \Phi_{02^{\prime}} .
$$


Applying the $[\bar{\delta}, D]$ commutator to $\left(-\Psi_{2}-\Phi_{11^{\prime}}+\Lambda\right)$ and using (NPc) [which tells us that $D \tau=(\varepsilon-\bar{\varepsilon}) \tau],(\mathrm{Bb})$ and $\Psi_{2} \neq 0$ one obtains $D \pi=-(\varepsilon-\bar{\varepsilon}) \pi$.

Similarly, from $(\mathrm{Bg})$ and the conjugate of $(\mathrm{Bj})$ one obtains

$$
\bar{\delta}\left(-\Psi_{2}-\Phi_{11^{\prime}}+\Lambda\right)=3 \pi \Psi_{2}-2 \bar{\tau} \Phi_{11}-\tau \Phi_{20^{\prime}} .
$$

Applying the commutator $[\bar{\delta}, \Delta]$ to $\left(-\Psi_{2}-\Phi_{11^{\prime}}+\Lambda\right)$, using (NPi) [which tells us $\Delta \pi=-(\gamma-\bar{\gamma}) \pi],(\mathrm{Bc})$ and $\Psi_{2} \neq 0$ one obtains $\Delta \tau=(\gamma-\bar{\gamma}) \tau$.

Thus from (NPc), (NPi), and the arguments above:

$$
\begin{aligned}
& D \pi=-(\varepsilon-\bar{\varepsilon}) \pi, \quad \Delta \tau=(\gamma-\bar{\gamma}) \tau \\
& D \tau=(\varepsilon-\bar{\varepsilon}) \tau, \quad \Delta \pi=-(\gamma-\bar{\gamma}) \pi .
\end{aligned}
$$

Note that to arrive at (2.5) in conformally flat cases one would need to derive it by a different argument.

It will now be shown that a position-dependent rotation (the remaining frame freedom) can always be used to achieve

$$
\varepsilon=\bar{\varepsilon} \text { and } \gamma=\bar{\gamma}
$$

and hence that

$$
D \Phi_{02^{\prime}}=\Delta \Phi_{02^{\prime}}=D \pi=\Delta \pi=D \tau=\Delta \tau=0 .
$$

For non-zero $\tau$ and $\pi$, if either $\pi$ or $\tau$ is real (2.8) follows immediately from (2.5)-(2.6). If $\pi$ and $\tau$ are both non-zero $D(\ln (\tau / \pi))=2(\varepsilon-\bar{\varepsilon})$ and $\Delta(\ln (\tau / \pi))=$ $2(\gamma-\bar{\gamma})$. If then $\tau / \pi$ is real, these imply (2.7). Under a rotation through an angle $\theta$, $(\varepsilon-\bar{\varepsilon})^{\star}=(\varepsilon-\bar{\varepsilon})+i D \theta$ and $(\gamma-\bar{\gamma})^{\star}=(\gamma-\bar{\gamma})+i \Delta \theta$. Hence if $\tau / \pi$ is not real, $\theta=\frac{1}{2} \operatorname{Im}(\ln (\pi / \tau))$ achieves (2.7). If just one of $\pi$ or $\tau$ is nonzero, its argument gives a suitable $\theta$. If $\pi=0=\tau$ one needs to show that the equations

$$
i D \theta=-(\varepsilon-\bar{\varepsilon}) \text { and } i \Delta \theta=-(\gamma-\bar{\gamma}) \text {, }
$$

are compatible. This is done by applying the $[\Delta, D]$ commutator to $\theta$. Note that (NPh) implies that $\Psi_{2}$ is real in this case; the imaginary part of (NPf) then shows that the two equations for $\theta$ are indeed compatible.

Finally, (NPe) and the complex conjugate of (NPd), and (NPr) and the complex conjugate of (NPo), are used to obtain

$$
D(\alpha-\bar{\beta})=0=\Delta(\alpha-\bar{\beta})
$$

Inserting these results into the higher derivatives, using CLASSI, shows that the second, third and fourth derivatives of the Riemann tensor are also boost invariant. This will also be true in conformally flat spacetimes if (2.1), (2.10) and (2.8) hold, but in the case studied in Sect. $3.4\left(\mathrm{~A}_{3}\right)$ is required to establish (2.8). 
One can show, as in the LRS case, that in Petrov type D one cannot have $s=1$ and $t_{2}=1, t_{1}=t_{0}=0$ which would require $\left(\mathrm{A}_{4}\right)$ to be checked. To eliminate the possibility, the calculations follow a similar logic to those in MacCallum [9]. Necessarily $\Psi_{2} \neq 0$, and $\Phi_{11^{\prime}}$ and $\Phi_{02^{\prime}}$ would be constant. That $\nabla \Psi_{A B^{\prime}}$ is constant implies $\tau$ and $\pi$ are constant. If at least one of them is nonzero, then (NPg) and/or (NPp) imply that $\bar{\alpha}-\beta$ is constant (possibly zero). Direct calculation (using CLASSI) then shows $t_{2}=0$ i.e. all terms in the second derivatives are also constant, so the CartanKarlhede procedure terminates. If both $\pi$ and $\tau$ are zero, $(\mathrm{NPg})$ implies $\Phi_{02^{\prime}}=0$, and then inspection (using CLASSI) shows that all first derivatives of the Riemann tensor, and hence all higher derivatives, are zero, and the Cartan-Karlhede procedure terminates at step 1.

Thus for LBS Petrov type D spacetimes it is sufficient to check $\left(\mathrm{A}_{3}\right)$ and the Cartan-Karlhede procedure must terminate at the third step or earlier. This proves the following.

Theorem 2 If a spacetime of Petrov type D is such that the Riemann tensor and its first derivative are invariant under a local boost invariance, then the spacetime is locally boost symmetric and admits a local isometry group $G_{r}(r \geq 3)$.

The converse of Theorem 2 is obvious, and by the equivalence shown above this proves Theorem 1. Note that as in Section 3 of MacCallum [9] the invariance of the derivatives of the Ricci tensor has not been used to derive the results, only checked, and $\Psi_{2} \neq 0$ was used only in deriving $\left(\mathrm{C}^{\dagger}\right)$ and (2.5). The LBS conclusion depends only on (2.1), (2.10) and (2.8).

In the following section it is shown that Theorem 2 is still true with 'Petrov type D' replaced by 'conformally flat', unless the Ricci tensor is of Segre type [1(11,1)] when some cases require LBI of the curvature and its first three derivatives to ensure LBS.

\section{Conformally flat spacetimes with local boost invariance}

The conformally flat cases to be considered are those Ricci tensor types appearing in Table $1 .\left(\mathrm{A}_{m}\right)$ is assumed to hold with a group $g$ which contains a boost. By the same argument as in MacCallum [9], Ricci tensors of Segre type [(111,1)] are easily disposed of: the spacetimes are of constant curvature, the subgroup $g$ in $\left(\mathrm{A}_{m}\right)$ is the trivial one comprising the whole Lorentz group, $s=6, t_{p}=0=t_{0}$, and there is a group $G_{10}$ transitive on the whole spacetime. The Cartan-Karlhede procedure terminates at the first step and $\left(\mathrm{A}_{0}\right)$ suffices because it will imply $\left(\mathrm{A}_{1}\right)$.

In the rest of this section the actual $\left(\mathrm{A}_{m}\right)$ required for local LBS in conformally flat spacetimes with the various Ricci tensors which admit a boost invariance, but are of less symmetry than Segre type [(111,1)], are studied.

\subsection{The first derivatives and Bianchi identities}

The first step is to impose LBI on the first derivatives of $\Phi$ and $\Lambda$. Then one can try to derive $\left(\mathrm{C}^{\dagger}\right)$, which were obtained in Petrov type D cases from invariance of $\nabla \Psi$, and look for an appropriate variant of the rest of the arguments in Sect. 2. 
With LBI of the curvature only $\Lambda, \Phi_{11^{\prime}}$ and $\Phi_{02^{\prime}}=\overline{\Phi_{20^{\prime}}}$ can be nonzero components in a suitable canonically chosen frame, and since $\Phi_{11^{\prime}}$ and $\Lambda$ are invariant under the remaining allowed changes of frame, $\left(\mathrm{A}_{1}\right)$ requires that $\Delta \Phi_{11^{\prime}}=D \Phi_{11^{\prime}}=\Delta \Lambda=$ $D \Lambda=0$. (Note that the invariance of curvature is being assumed here, rather than $\Phi_{00^{\prime}}=\Psi_{22^{\prime}}=0$ being deduced from other assumptions as in Sect. 2.) The terms in $\nabla \Phi$ that must vanish if $\left(\mathrm{A}_{1}\right)$ holds are

$$
\begin{aligned}
3 \nabla \Phi_{01^{\prime}} & =2\left(2 \kappa \Phi_{11^{\prime}}+\bar{\kappa} \Phi_{02^{\prime}}\right), \\
3 \nabla \Phi_{02^{\prime}} & =D \Phi_{02^{\prime}}+2(\bar{\rho}-\varepsilon+\bar{\varepsilon}) \Phi_{02^{\prime}}+4 \sigma \Phi_{11^{\prime}}, \\
9 \nabla \Phi_{11^{\prime}} / 2 & =2(\rho+\bar{\rho}) \Phi_{11^{\prime}}+\sigma \Phi_{20^{\prime}}+\bar{\sigma} \Phi_{02^{\prime}}, \\
3 \nabla \Phi_{13^{\prime}} & =\Delta \Phi_{02^{\prime}}+2(\bar{\gamma}-\gamma-\mu) \Phi_{02^{\prime}}-4 \bar{\lambda} \Phi_{11^{\prime}}, \\
9 \nabla \Phi_{22^{\prime}} / 2 & =-2(\mu+\bar{\mu}) \Phi_{11^{\prime}}-\lambda \Phi_{02}-\bar{\lambda} \Phi_{20^{\prime}} . \\
3 \nabla \Phi_{23^{\prime}} & =-2\left(\nu \Phi_{02^{\prime}}+2 \bar{\nu} \Phi_{11^{\prime}}\right) .
\end{aligned}
$$

In addition $\nabla \Phi_{00^{\prime}}=\nabla \Phi_{33^{\prime}} \equiv 0$ here. Of the above equations, (3.1) is equivalent to $(\mathrm{Ba}),(3.3)$ to $(\mathrm{Bi})$ or the real part of $(\mathrm{Be}),(3.5)$ to $(\mathrm{Bk})$ or the real part of $(\mathrm{Bf})$, and (3.6) to the conjugate of $(\mathrm{Bd})$. The Bianchi identities $(\mathrm{Be})$ and $(\mathrm{Bf})$ give

$$
\bar{\sigma} \Phi_{02^{\prime}}+2 \rho \Phi_{11^{\prime}}=0, \text { and } \bar{\lambda} \Phi_{20^{\prime}}+2 \mu \Phi_{11^{\prime}}=0 .
$$

In the general case the only information from boost invariance of $\nabla \Phi$ additional to that in the Bianchi identities comes from (3.2) and (Bb), and (3.4) and the conjugate of $(\mathrm{Bc})$, which give respectively.

$$
\bar{\rho} \Phi_{02^{\prime}}+2 \sigma \Phi_{11^{\prime}}=0, \text { and } \bar{\mu} \Phi_{20^{\prime}}+2 \lambda \Phi_{11^{\prime}}=0 .
$$

If (3.8) is satisfied the first derivatives of the curvature are boost invariant. (In the special case of Segre type $[1(11,1)]$, where $D \Phi_{02^{\prime}}=\Delta \Phi_{02^{\prime}}=0,(3.8)$ is equivalent to $(\mathrm{Bb})$ and $(\mathrm{Bc})$, and contains no additional information. This case is considered in Sect. 3.4 below.)

The Bianchi identities $(\mathrm{Bg})$ and $(\mathrm{Bh})$ give

$$
2(\bar{\pi}+\tau) \Phi_{11^{\prime}}+(\pi+\bar{\tau}) \Phi_{02^{\prime}}=0 .
$$

The remaining information in the Bianchi identities is

$$
\begin{aligned}
& B g: \delta \Phi_{20^{\prime}}-2 \bar{\delta} \Lambda=(2 \bar{\alpha}-2 \beta-\bar{\pi}) \Phi_{20^{\prime}}-2 \pi \Phi_{11^{\prime}}, \\
& B j:-\delta \Phi_{11^{\prime}}-\bar{\delta} \Phi_{02^{\prime}}+3 \delta \Lambda=(-2 \alpha+2 \bar{\beta}+\pi-\bar{\tau}) \Phi_{02^{\prime}} \\
& \quad+2(\bar{\pi}-\tau) \Phi_{11^{\prime}} .
\end{aligned}
$$

\subsection{Ricci tensors of Segre type $[11(1,1)]$}

If such spacetimes obey $\left(\mathrm{A}_{\infty}\right)$ the Cartan-Karlhede procedure must terminate after at most 3 steps since $s=1$ at every step and at most two steps are needed to increase 
$t_{p}$ to the maximum of 2 . So $\left(\mathrm{A}_{3}\right)$ would suffice. As shown next, only $\left(\mathrm{A}_{1}\right)$ is actually required. All the resulting spacetimes admit a $G_{r}, r \geq 3$ acting on submanifolds of dimension at least 2 .

The ratio $\Phi_{02^{\prime}} / \Phi_{11^{\prime}}=2 c$ is fixed under the remaining frame freedom but may be position-dependent. (Using the remaining rotational freedom one could make $\Phi_{02^{\prime}}$ real and positive, and $c$ real, but this freedom may be needed as in Sect. 2 to obtain a frame in which (2.7) holds, using (2.5)-(2.6).) One must have $|c| \neq 1$ or the Segre type will be [1(11,1)]. Relations of the form $2 \Phi_{11^{\prime}} Q+\Phi_{02^{\prime}} \bar{Q}=0$ imply $Q=0$, since otherwise $|Q / \bar{Q}|=1 \neq|c|=\left|\Phi_{02^{\prime}} / \Phi_{11^{\prime}}\right|$ (If $|c|=1$ at some isolated points, continuity implies $Q=0$ there too.) Hence from (Ba) and ( $\mathrm{Bd}), \kappa=v=0$, and from (3.8) and (3.7) $\rho=\mu=\sigma=\lambda=0$. So (C1 ${ }^{\dagger}$ ) holds. In addition, (3.9) implies $\bar{\pi}+\tau=0$.

Equation (2.6) holds (being just (NPc) and (NPi)) and (2.5) then follows simply from it, using $\bar{\pi}+\tau=0$. One can then obtain (2.7) and (2.8), and complete the proof that $\left(\mathrm{A}_{1}\right)$ is sufficient to imply LBS in this case as in Sect. 2. One may note that since $D \Phi_{02^{\prime}}=\Delta \Phi_{02^{\prime}}=0, c$ is constant in the timelike two-planes determined by the boost.

\subsection{Ricci tensors of Segre type $[(11)(1,1)]$}

This Segre type is that of the Ricci tensor of a non-null electromagnetic field. Here $s_{0}=2$ and in a canonical frame $\Phi_{02^{\prime}}=0$. Assuming $\Phi_{11^{\prime}} \neq 0,(\mathrm{Ba})-(\mathrm{Bd})$ give

$$
\kappa=\sigma=\lambda=\nu=0 .
$$

If $s=2$ one must have $t_{1}=0$ and so the Cartan-Karlhede procedure terminates at step 1 since neither $s$ nor $t$ has changed and as in Section 3 of MacCallum [9] this gives the Bertotti-Robinson type solutions with a $G_{6}$ transitive on the whole spacetime, and $\left(\mathrm{A}_{1}\right)$ suffices.

For $s=1$ with $\hat{I}_{1}$ consisting of the boosts, $D \Lambda=D \Phi_{11^{\prime}}=\Delta \Lambda=\Delta \Phi_{11^{\prime}}=0$ and from (Be) and (Bf) $\rho=\mu=0$ so the conditions of Theorem 1 hold. As in the previous subsection, one has $\tau+\bar{\pi}=0$, so the proof that $\left(\mathrm{A}_{1}\right)$ with a one-dimensional group of boosts implies LBS proceeds as in Sect. 2.

\subsection{Ricci tensors of Segre type $[1(11,1)]$}

Here the invariance group is $\mathrm{SO}(2,1)$, generated by null rotations about $\boldsymbol{k}$ and about $\boldsymbol{l}$ and a boost in the $(\boldsymbol{k}, \boldsymbol{l})$ plane. The $\mathrm{SO}(2,1)$ group acts in a hyperplane and leaves invariant one direction in the $(\boldsymbol{m}, \overline{\boldsymbol{m}})$ plane. The Ricci tensor represents a tachyonic fluid, and a canonical form for it which is manifestly null rotation invariant (as in MacCallum [8]) about each of the null directions has only $\Phi_{11^{\prime}}$ and $\Phi_{02^{\prime}}$ non-zero with $2\left|\Phi_{11^{\prime}}\right|=\left|\Phi_{02^{\prime}}\right|$. (This is a specialization of the form for Segre type [11(1,1)], treated above.) Using the remaining freedom of spatial rotation in the $(\boldsymbol{m}, \overline{\boldsymbol{m}})$ plane one can set $\Phi_{02^{\prime}}=2 \Phi_{11^{\prime}}$ : the parameters of both null rotations are then pure imaginary and the vector orthogonal to the hyperplane in which the $\operatorname{SO}(2,1)$ acts is in the direction $\boldsymbol{m}+\overline{\boldsymbol{m}}$. 
The boost rescales the parameters of the null rotations. One might therefore have invariance under a two-dimensional subgroup of $\mathrm{SO}(2,1)$ generated by the boost and one of the null rotations (see entry R6 in Table 6.1 of Hall [6]). Among the quantities defined by MacCallum and Åman [10], only $\nabla^{k} \Phi_{A B^{\prime}}$ and $\nabla^{k} \Lambda_{A B^{\prime}}$, both of which are Hermitian, and d'Alembertians thereof, have to be considered. Boost invariance implies that of the components of $\nabla^{k} \Phi_{A B^{\prime}}$, only those with $A+B=2+k$ can be nonzero. If then $\nabla^{k} \Phi_{A B^{\prime}}$ is invariant under one of the null rotations, then from the Hermitian symmetry it will also be invariant under the other. Thus $\nabla^{k} \Phi_{A B^{\prime}}$ will be $\mathrm{SO}(2,1)$ invariant. The same applies to $\nabla^{k} \Lambda_{A B^{\prime}}$ and the d'Alembertians of spinors of lower derivative order.

So $s=2$ is impossible and either $s=3$ or $s=1$. If $s=3$ there can be at most one independent function of position (as there is just one spacelike direction fixed under $g$ ). The spacetime admits a $G_{6}$ (or in special cases a $G_{7}$, cf. Rebouças and Teixeira [12]) acting on timelike hypersurfaces of constant curvature. The metrics include analogues of the FLRW metrics for perfect fluids.

The remaining case is where $s=1$ and $g$ in $\left(\mathrm{A}_{m}\right)$ is just the boost invariance ${ }^{2}$. From $(\mathrm{Ba})$ and $(\mathrm{Bd})-(\mathrm{Bf})$ one has

$$
\kappa+\bar{\kappa}=v+\bar{v}=\bar{\mu}+\lambda=\bar{\rho}+\sigma=0,
$$

and then $(\mathrm{Bb})$ and $(\mathrm{Bc})$ give (2.7). In this case the Bianchi identities and $D \Phi_{11^{\prime}}=$ $\Delta \Phi_{11^{\prime}}=D \Lambda=\Delta \Lambda=0$ ensure $\left(\mathrm{A}_{1}\right),(3.8)$ being equivalent to (3.7) in this case. From (3.9) one has

$$
(\pi+\bar{\pi})+(\tau+\bar{\tau})=0 .
$$

The real parts of the left sides of (3.10) and (3.11) are proportional to the real part of $\delta\left(\Phi_{11^{\prime}}-\Lambda\right)$. For the right sides to be compatible one must have

$$
0=(\alpha+\bar{\alpha})-(\beta+\bar{\beta})+(\pi+\bar{\pi})
$$

Eliminating between the imaginary parts of (3.10) and (3.11) yields

$$
\begin{aligned}
(\delta-\bar{\delta}) \Lambda & =[(\bar{\alpha}-\beta)-(\alpha-\bar{\beta})] \Phi_{11^{\prime}}, \\
(\delta-\bar{\delta}) \Phi_{11^{\prime}} & =[(\bar{\alpha}-\beta)-(\alpha-\bar{\beta})] \Phi_{11^{\prime}}
\end{aligned}
$$

The perhaps surprising equality of the right sides does not imply that $\delta\left(\Phi_{11^{\prime}}-\Lambda\right)=0$.

The real parts of (NPc) and (NPi) give $D(\tau+\bar{\tau})=0=\Delta(\pi+\bar{\pi})$, so

$$
D(\tau+\bar{\tau})=\Delta(\pi+\bar{\pi})=\Delta(\tau+\bar{\tau})=D(\pi+\bar{\pi})=0 .
$$

\footnotetext{
2 It might be convenient to carry out the necessary calculations using the $3+1$ orthonormal tetrad formalism based on a spacelike congruence introduced by Harness [7].
} 
From the imaginary part of $(\mathrm{NPg})$ minus the conjugate of $(\mathrm{NPh})$, using (3.12) and (3.14),

$$
\delta(\pi+\bar{\pi})=\bar{\delta}(\pi+\bar{\pi})
$$

From the conjugate of (NPd) together with (NPe), and from the conjugate of (NPr) with (NPo), one finds using (3.14) that

$$
D(\bar{\alpha}-\beta)=\Delta(\bar{\alpha}-\beta)=0
$$

which is consistent with (3.14) and (3.17).

From $(\mathrm{NPb})$ and the complex conjugate of $(\mathrm{NPa})$, and from the conjugate of $(\mathrm{NPj})$ and (NPn), one obtains

$$
\kappa(2 \bar{\alpha}-2 \beta+(\pi+\bar{\pi}))=0=v(2 \bar{\alpha}-2 \beta+(\pi+\bar{\pi})) .
$$

Since $\kappa$ and $v$ are pure imaginary, the imaginary parts of these equations follow from (3.14) while the real parts give $\kappa \Im_{\alpha \beta}=\nu \mathfrak{\Im}_{\alpha \beta}=0$, where $\mathfrak{\Im}_{\alpha \beta} \equiv(\alpha-\bar{\beta})-(\bar{\alpha}-$ $\beta$ ). (From the relations between null and orthonormal tetrads obtainable using and Wainwright [4] and Harness [7] one finds that $\mathfrak{\Im}_{\alpha \beta}$ plays the same rôle here as $\dot{u}_{1}$ did in the conformally flat perfect fluids studied in MacCallum [9].) So far only $\left(\mathrm{A}_{1}\right)$ has been used.

Calculating $\nabla^{2} \Phi$ shows that the $00^{\prime}, 01^{\prime}, 34^{\prime}$ and $44^{\prime}$ components are identically zero. Most of the other components with $A+B \neq 4$ vanish on simplification with the help of (3.16), (3.13), (3.14), and (3.20), which all follow from $\left(\mathrm{A}_{1}\right)$. The remaining components which must vanish for $\operatorname{LBI}\left(\nabla^{2} \Phi_{03}\right.$ and $\left.\nabla^{2} \Phi_{14^{\prime}}\right)$ yield $\Im_{\alpha \beta} \sigma=\Im_{\alpha \beta} \mu=0$. For the spacetime to obey $\left(\mathrm{A}_{2}\right)$ thus requires $\mathfrak{\Im}_{\alpha \beta}$ multiplying each of $\kappa, \sigma, \mu$ and $v$ to be zero.

To satisfy $\left(\mathrm{A}_{2}\right)$ there are now two possibilities: either (a) $\mathfrak{\Im}_{\alpha \beta}=(\delta-\bar{\delta}) \Phi_{11^{\prime}}=$ $(\delta-\bar{\delta}) \Lambda=0$, showing that $\Phi_{11^{\prime}}$ and $\Lambda$ are constant in the hyperplanes defined by the $\mathrm{SO}(2,1)$ action, or (b) $\mathfrak{\Im}_{\alpha \beta} \neq 0$ and $\left(\mathrm{C} 1^{\dagger}\right)$ holds.

In case (a), we find that $\nabla^{k} \Phi$ and $\nabla^{k} \Lambda$ are invariant under a null rotation about $\boldsymbol{k}$ for $k=1 \ldots 3$ and therefore $s=3$ by the earlier argument that $s_{i}$ cannot be 2 . We thus again have at least a $G_{6}$ if $\Im_{\alpha \beta}=0$. Note that $\left(\mathrm{A}_{2}\right)$ was checked but does not give extra conditions in this case.

In case (b), one now has the conditions $\left(\mathrm{Cl}^{\dagger}\right)$ and (2.7). (NPc) and (NPi) give $D \tau=\Delta \pi=0$. To complete (2.8), one has to show that $D \pi=\Delta \tau=0$. Then the curvature derivatives up to the fourth will have boost invariance, as in Sect. 2. (Note that since $(\delta-\bar{\delta}) \Phi_{11^{\prime}} \neq 0, t_{0}=0$ need not be considered here.) One finds $\nabla^{3} \Phi_{22^{\prime}}=-6 \Phi_{11^{\prime}} \Im_{\alpha \beta} D(\pi+\tau) / 25$ and $\nabla^{3} \Phi_{33^{\prime}}$ gives the same with $\Delta$ replacing $D$. Thus to obtain $(2.8)$ one needs $\left(\mathrm{A}_{3}\right)$ in this case. 


\section{Conclusion}

The work in Sects. 2 and 3 gives the following analogue of Theorem 3 of MacCallum [9].

Theorem 3 In spacetimes with a Ricci tensor of Segre type $[1(11,1)]$ whose distinguished spacelike eigenvector is not geodesic, local boost invariance of the curvature and its derivatives up to the third holds if and only if the spacetime is locally boost symmetric. In all other cases, local boost invariance of the curvature and its first derivatives holds if and only if the spacetime is locally boost symmetric.

Acknowledgements I am grateful to Jan Åman for his work in developing the software CLASSI and to Filipe Mena, who was kind enough to check and correct some of the calculations.

Conflict of interest The author is an Editorial Board member of the journal General Relativity and Gravitation.

Open Access This article is licensed under a Creative Commons Attribution 4.0 International License, which permits use, sharing, adaptation, distribution and reproduction in any medium or format, as long as you give appropriate credit to the original author(s) and the source, provide a link to the Creative Commons licence, and indicate if changes were made. The images or other third party material in this article are included in the article's Creative Commons licence, unless indicated otherwise in a credit line to the material. If material is not included in the article's Creative Commons licence and your intended use is not permitted by statutory regulation or exceeds the permitted use, you will need to obtain permission directly from the copyright holder. To view a copy of this licence, visit http://creativecommons.org/licenses/by/4.0/.

\section{References}

1. Cahen, M., Defrise, L.: Lorentzian 4-dimensional manifolds with local isotropy. Commun. Math. Phys. 11, 56 (1968)

2. Ellis, G.F.R.: Dynamics of pressure-free matter in general relativity. J. Math. Phys. 8, 1171 (1967)

3. Geroch, R., Held, A., Penrose, R.: A space-time calculus based on pairs of null directions. J. Math. Phys. 14, 874 (1973)

4. Goode, S.W., Wainwright, J.: Characterization of locally rotationally symmetric space-times. Gen. Relativ. Gravit. 18, 315 (1986)

5. Hall, G.S.: The global extension of local symmetries in general relativity. Class. Quant. Grav. 6(2), 157-161 (1989)

6. Hall, G.S.: Symmetries and Curvature Structure in General Relativity, World Scientific Lecture Notes in Physics, vol. 26. World Scientific, Singapore (2004)

7. Harness, R.S.: Space-times homogeneous on a time-like hypersurface. J. Phys. A 15(1), 135-151 (1982)

8. MacCallum, M.A.H.: Totally symmetrized spinors and null rotation invariance. Class. Quant. Grav. 37, 195011 (2020)

9. MacCallum, M.A.H.: Spacetimes with continuous linear isotropies I: spatial rotations. Gen. Relativ. Gravit. 53, 57 (2021)

10. MacCallum, M.A.H., Åman, J.E.: Algebraically independent $n$-th derivatives of the Riemannian curvature spinor in a general spacetime. Class. Quant. Grav. 3(6), 1133-1141 (1986)

11. McNutt, D., Coley, A., Wylleman, L., Hervik, S.: Locally boost isotropic spacetimes and the type $\mathbf{D}^{\mathbf{k}}$ condition (2019). arXiv:1907.08957

12. Rebouças, M.J., Teixeira, A.F.F.: Homogeneous space-times with seven isometries. J. Math. Phys. 33, 2855-2862 (1992)

13. Siklos, S.T.C.: Singularities, invariants and cosmology, Ph.D. thesis, Cambridge (1976)

14. Stephani, H., Kramer, D., MacCallum, M.A.H., Hoenselaers, C.A., Herlt, E.: Exact solutions of Einsteins field equations, 2nd edition, Cambridge University Press. Corrected Paperback edition, 2009, Cambridge (2003) 
15. Stewart, J.M., Ellis, G.F.R.: On solutions of Einsteins equations for a fluid which exhibit local rotational symmetry. J. Math. Phys. 9, 1072 (1968)

Publisher's Note Springer Nature remains neutral with regard to jurisdictional claims in published maps and institutional affiliations. 